\title{
Educating to inspire active learning approaches in mathematics in Norwegian universities
}

\author{
K. Bjørkestøl, ${ }^{1}$ I.C. Borge, ${ }^{2}$ S. Goodchild, ${ }^{1}$ H.K. Nilsen, ${ }^{1}$ O.H.M. Tonheim, ${ }^{3}$ \\ ${ }^{I}$ University of Agder, ${ }^{2}$ University of Oslo, ${ }^{3}$ Volda University College
}

\begin{abstract}
This is a report of an analysis of some of the data generated by a national survey of teaching approaches used in higher education mathematics courses. The overall purpose of the survey was to explore how widespread is the use of teaching approaches that might promote students' active learning of mathematics. The paper includes a brief presentation of the authors meaning of the expression "teaching actions that have the potential to promote active learning". The analysis focuses on the responses of 95 lecturers working in 13 Norwegian HE institutions. The goal is to expose underlying patterns in lecturers' responses to questions about the teaching actions they may incorporate in their practice. The analysis incorporates descriptive statistics (e.g., mean scores) and exploratory factor analysis to expose underlying reasons for patterns of lecturers' responses. Qualitative, interpretative approaches are used, both in the design of the survey instrument and in making sense of the outcome from the statistical analysis.
\end{abstract}

\section{INTRODUCTION}

We report from our analysis of a national survey of teaching approaches adopted in mathematics courses in Norwegian Higher Education Institutions (HEIs). The principal goal of the survey was to gain an insight into the use of teaching approaches that were specifically designed to stimulate students' active learning in mathematics in Norwegian HEIs. The survey was conducted as a joint action by The Norwegian Mathematics Council (NMR) and MatRIC, Centre for Research, Innovation and Coordination of Mathematics Teaching. A "delphi study" was used to develop a questionnaire focusing on mathematics teaching and learning. The development of the survey instrument was reported at the 2019 MNT conference in Tromsø. The survey instrument was distributed online to lecturers and students through the institutional representatives of NMR around the end of 2019 and beginning of 2020. This presentation is based on analysis of responses from 95 lecturers that completed the questionnaire. These lecturers represent 13 HEIs. The survey also collected responses from 1863 students (9 HEIs), and 98 student learning assistants (6 HEIs), (analyses of these results is forthcoming).

A preliminary report, mainly of a descriptive nature, was presented at the annual meeting of NMR in September 2020. This report is available at https://www.matric.no/resources/138. In this presentation we report from an analysis of lecturers' responses to questions about the types of teaching actions they use. Both qualitative interpretation and statistical relationships are used. Qualitatively we draw attention to teaching approaches that may inspire students' active learning, e.g., communication and working methods, this was already embedded in the design of the survey instrument. Quantitatively we analyse the data generated by the survey to expose relationships between teaching approaches that may be related to encouraging students' active (cognitive) engagement in their learning.

This is the first national study related to active learning in mathematics in Norwegian HEIs. However, it builds on work that has been reported in the USA in which active learning approaches have been associated with improved student performance (Freeman et al. 2014, Apkarian 2020, Rasmussen et al. 2019). At the 2019 MNT conference a survey of active learning approaches in the biology at University of Bergen was reported (Enberg et al. 2019). The survey we report adds other dimensions related to knowledge of practices in mathematics within Norwegian STEM education.

\section{ACTIVE LEARNING}

There is no common agreement about what is meant by the expression "active learning". Moreover, it is impossible to assert with any degree of certainty that active learning will occur in some teachinglearning contexts and not others. For the purposes of this presentation, we will assume that "active learning" occurs when students are engaged in learning tasks that require them to be cognitively (and 
metacognitively) active. They are engaged in processes of constructing meanings and understanding, of engaging conceptually with the subject matter in focus, making connections and critically appraising the sense they are forming.

This is consistent with Laursen and Rasmussen (2019), “... learning experiences that are rich and meaningful: centered on students' ideas, requiring their mental engagement in and out of class, and accountable to their prior understandings." (p. 129). By contrast, passive learning is a form of surface engagement that is founded on the belief or hope that something will be retained in memory.

Active learning can occur in formal traditional lectures in which the teacher stands at the front, writing on a chalkboard the mathematics to be learned. In this case the student needs to attend critically to the presentation and their concurrent understanding, in the context of their existing knowledge, which they accept could be fallible, and with the goal of making sense of the mathematics taught. On the other hand, in the same situation, a student may be so active, especially trying to copy down everything the teacher writes, that they are so busy producing their own record that they have no opportunity to think. Both students are active, one is cognitively active, the other physically, although in both the actions are under some form of mental control.

In some teaching/learning situations, it can be difficult for a student to displace cognitive and metacognitive activity by physical or other cognitively irrelevant activity; for example, in one-to-one problem-oriented discussion between student and teacher. Our focus is on teaching approaches that promote active learning, the key word is "promote", meaning the student is more likely to be prompted into cognitive activity focused on the learning material.

Consider the following different educational contexts: 1. Lectures to large groups of students, 2 . Seminars, 3. Students working in large groups, 4 . Students working in small groups. All of these contexts are educationally meaningful and can be effective in enabling students to learn. All of the contexts open the possibility for students to be cognitively and metacognitively focused and active on the subject matter being taught. However, it is less easy for a student to "hide" or be active in other ways than attending to the meaning of the material being taught in small group work (4), than for any of the other three contexts. Working in large groups can open the possibility of student collaboration that again makes it more difficult to be distracted. Consequently, it is possible to assert that working in small groups has a higher potential to promote active learning, whilst lectures and seminars have lower potential. Working in large groups lying somewhere between these two. We emphasise POTENTIAL TO PROMOTE active learning, in the sense defined above. We do not make claims about effectiveness or ineffectiveness of any educational context because there are actions a lecturer can take, for example, in a large lecture to raise the potential to promote active learning, such as the use of student response devices (clickers or mobile phone apps like Socrative and Kahoot) and actions such as "think, pair, share", etc. This presentation will not focus on group size.

\section{RESULTS AND ANALYSIS}

Table 1 is composed of statements about teaching actions used within the survey instrument. The items are arranged into three categories, colour coded: black (B), red (R), and green (G). Broadly, the items coded $\mathrm{B}$ relate to expectations about what students will do, items coloured $\mathrm{R}$ are about lecturers providing or identifying resources for students. Item i18, G, relates to the general availability of course resources. We do not evaluate the approach etc. as good or bad, nor indicate an opinion about salience/potential to promote active learning as explained above. Respondents were asked if they employed the action in their teaching and could choose a response from: Not relevant, Never, Seldom, Sometimes, Often, Always. These have been assigned values, 0 to 5 respectively, and means for the lecturers' responses to these statements are included in parentheses following each statement. In Table 1 items within each category are ranked according to the mean score for the item; this is not the same order as in the questionnaire. It appears that taking action to ensure students come to class prepared, expecting students to work in small groups, and students working on tasks individually are the most common approaches that are used which we judge also to have the potential to promote active learning.

To expose further patterns within the responses we explored correlations and then used factor analysis to look for the existence of underlying (latent) factors that might explain patterns within the data. Statistics from the factor analysis are reproduced below. 
Table 1. Salience/potential to promote active learning: Teaching actions (mean response in parentheses)

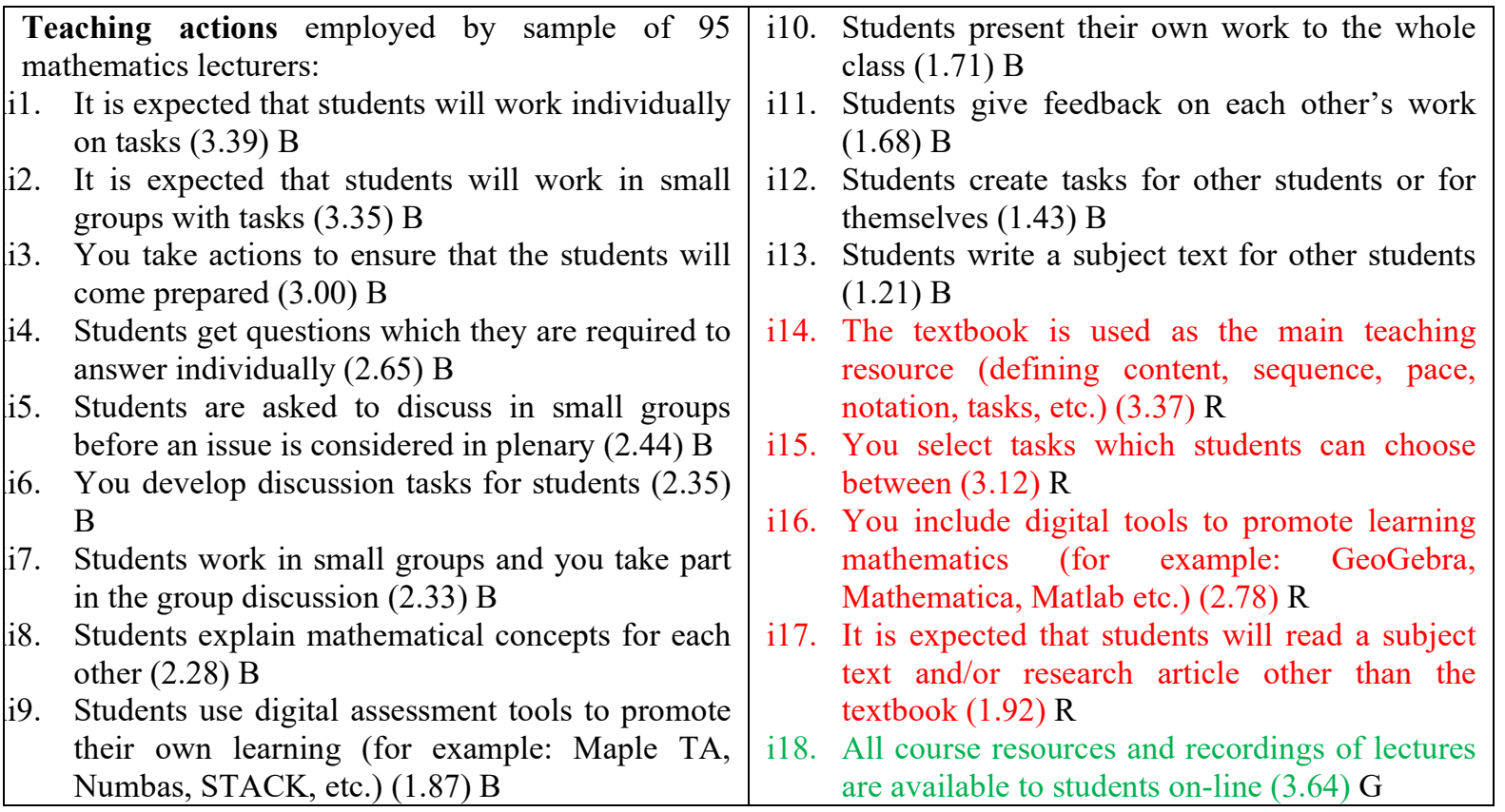

The mean scores inform about the types of actions employed (or not) in mathematics teaching, but alone they are insufficient to expose any underlying (latent) characteristic of teaching practice that may influence teaching approaches commonly used. To look for patterns and underlying relationships between different approaches, the correlations between the approaches were calculated; see Table 2 .

Table 2. Correlations (Kendall's tau) between teaching actions. Shaded cells show correlations larger than 0.5.

\begin{tabular}{|c|c|c|c|c|c|c|c|c|c|c|}
\hline Corr. & $\mathrm{i} 1$ & $\mathrm{i} 2$ & $\mathrm{i} 3$ & $\mathrm{i} 4$ & $\mathrm{i} 5$ & $\mathrm{i} 6$ & $\mathrm{i} 7$ & $\mathrm{i} 8$ & $\mathrm{i} 9$ & $\mathrm{i} 10$ \\
\hline $\mathrm{i} 1$ & 1.000 & & & & & & & & & \\
\hline $\mathrm{i} 2$ & 0.236 & 1.000 & & & & & & & & \\
\hline $\mathrm{i} 3$ & 0.152 & 0.252 & 1.000 & & & & & & & \\
\hline $\mathrm{i} 4$ & 0.110 & 0.189 & 0.232 & 1.000 & & & & & & \\
\hline $\mathrm{i} 5$ & -0.087 & 0.309 & 0.295 & 0.378 & 1.000 & & & & & \\
\hline $\mathrm{i} 6$ & -0.104 & 0.296 & 0.312 & 0.382 & 0.587 & 1.000 & & & & \\
\hline $\mathrm{i} 7$ & -0.001 & 0.340 & 0.280 & 0.370 & 0.460 & 0.565 & 1.000 & & & \\
\hline $\mathrm{i} 8$ & 0.050 & 0.257 & 0.267 & 0.293 & 0.428 & 0.468 & 0.474 & 1.000 & & \\
\hline $\mathrm{i} 9$ & 0.030 & 0.158 & 0.030 & 0.049 & 0.025 & 0.097 & -0.021 & -0.008 & 1.000 & \\
\hline $\mathrm{i} 10$ & -0.115 & 0.198 & 0.240 & 0.394 & 0.544 & 0.496 & 0.474 & 0.409 & 0.006 & 1.000 \\
\hline $\mathrm{i} 11$ & 0.079 & 0.258 & 0.198 & 0.370 & 0.434 & 0.557 & 0.520 & 0.504 & -0.003 & 0.447 \\
\hline $\mathrm{i} 12$ & 0.034 & 0.270 & 0.105 & 0.320 & 0.394 & 0.460 & 0.369 & 0.449 & 0.072 & 0.487 \\
\hline $\mathrm{i} 13$ & 0.025 & 0.200 & 0.138 & 0.311 & 0.401 & 0.441 & 0.366 & 0.442 & 0.111 & 0.520 \\
\hline $\mathrm{i} 14$ & 0.202 & 0.001 & -0.111 & -0.034 & -0.143 & -0.252 & -0.126 & -0.200 & 0.108 & -0.208 \\
\hline $\mathrm{i} 15$ & 0.169 & 0.124 & 0.132 & 0.267 & 0.123 & 0.115 & 0.096 & 0.151 & 0.160 & 0.081 \\
\hline $\mathrm{i} 16$ & -0.078 & 0.132 & 0.066 & 0.254 & 0.196 & 0.270 & 0.222 & 0.084 & 0.323 & 0.128 \\
\hline $\mathrm{i} 17$ & 0.027 & 0.148 & 0.145 & 0.310 & 0.432 & 0.512 & 0.459 & 0.346 & 0.092 & 0.480 \\
\hline $\mathrm{i} 18$ & 0.032 & 0.130 & -0.056 & 0.026 & -0.028 & 0.084 & 0.077 & -0.073 & -0.009 & 0.006 \\
\hline
\end{tabular}

Table 2. (continued). Correlations (Kendall's tau) between teaching actions. Correlations larger than 0.5 shaded.

\begin{tabular}{|c|c|c|c|c|c|c|c|c|}
\hline & $\mathrm{i} 11$ & $\mathrm{i} 12$ & $\mathrm{i} 13$ & $\mathrm{i} 14$ & $\mathrm{i} 15$ & $\mathrm{i} 16$ & $\mathrm{i} 17$ & $\mathrm{i} 18$ \\
\hline $\mathrm{i} 11$ & 1.000 & & & & & & & \\
\hline $\mathrm{i} 12$ & 0.664 & 1.000 & & & & & & \\
\hline $\mathrm{i} 13$ & 0.530 & 0.644 & 1.000 & & & & & \\
\hline $\mathrm{i} 14$ & -0.188 & -0.189 & -0.215 & 1.000 & & & & \\
\hline $\mathrm{i} 15$ & 0.138 & 0.048 & 0.140 & 0.284 & 1.000 & & & \\
\hline $\mathrm{i} 16$ & 0.166 & 0.245 & 0.201 & 0.019 & 0.170 & 1.000 & & \\
\hline $\mathrm{i} 17$ & 0.529 & 0.575 & 0.534 & -0.204 & -0.005 & 0.196 & 1.000 & \\
\hline $\mathrm{i} 18$ & 0.058 & 0.043 & 0.114 & -0.024 & 0.056 & 0.113 & 0.061 & 1.000 \\
\hline
\end{tabular}


(Note: Kendall's tau is used because this is often recommended for ordered data). It can be observed that Table 2 reveals that high correlations mostly occur with items with similar means, because of the way items are ordered and labelled, these occur close to the diagonal of the correlation matrix. The correlations suggest there may be evidence of broad teaching approaches employed across the cohort from which the data were generated, but alone they are insufficient to point to underlying characteristics or latent factors. To expose these, it is necessary to explore more deeply the patterns across correlations.

With few responses from teachers $(n=95)$ it is not possible to draw definite conclusions and further statistical analysis is conducted only as a means of developing a feeling for patterns that might emerge from a larger, representative sample of the population. With this spirit of exploration in mind we explored the data using exploratory factor analysis (EFA), to get a sense of the number of latent factors. In factor analysis we try to identify items that react on (load onto) the latent factors in similar ways. High loadings indicate strong relationship like coefficients in regression analysis. We reproduce these analyses below. Very simply, EFA is a digital, iterative approach to finding underlying (latent) factors that optimally explain (or produce) the total variation in the variance-covariance matrix of the item scores. This is carried out here by the robust method called weighted least squares.

Analysis of eigenvalues (four greatest: $5.84,1.16,0.85,0.73$ ) and subsequent parallel analysis suggested models with 2 to 4 factors. Exploratory Factor Analyses based on a model with 2 factors, weighted least squares method and oblique rotation, results in a correlation of 0.28 between the factors. The factors are plotted in Figure 1.

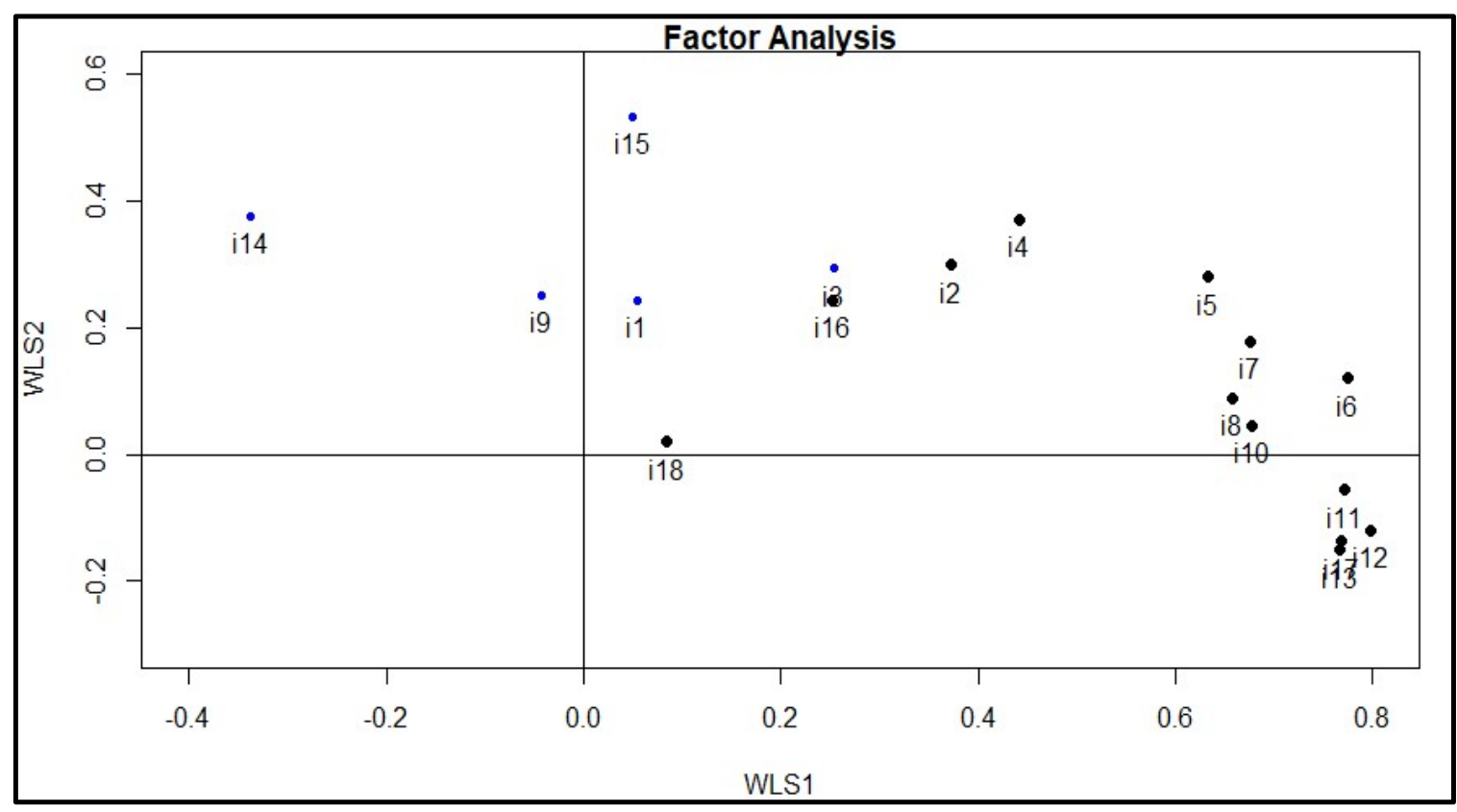

Fig. 1. Item/statement loadings on a two-factor model.

Table 3. shows the item loadings for two-, three- and four-factor models. Items i5-i8, and i10 have high loadings on the first factor in all models explored. We note that a common element in these items is that students must communicate actively, the means (except for i10) are mid-range (2.44-2.28) and the partial correlations are larger than 0.4. Items i11-i13, with smallest means in the group of items that focus on what students will do, include also high partial correlations, but the three EFA models are inconsistent about the items that have high loadings on their relevant factors. None of the teaching actions that we categorise as red or green has high partial correlations, and their highest loadings vary between the factors in the three models. Notice the behaviour of item i17 across the models - aligning with items i11-i13, where both correlations and loadings differ from the (qualitatively asserted) red or green items. It appears that items i11-i13 and i17 relate to an expectation that students will be independent learners. The highest correlation between the factors (See Table 4.) is between WLS1 and WLS2 in the three-factor model. This may reflect that i11-i13 are more strongly connected to the first factor in both the two-factor and four-factor models. Because of the small sample, we have not gone further by using Confirmatory Factor Analysis to make a more precise identification of latent factors. 
Table 3. Statement/item loadings for two- and three-factor EFA models

\begin{tabular}{|c|c|c|c|c|c|c|c|c|c|}
\hline & \multicolumn{2}{|c|}{ Two-factors } & \multicolumn{3}{|c|}{ Three-factors } & \multicolumn{4}{|c|}{ Four-factors } \\
\hline & WLS1 & WLS2 & WLS1 & WLS2 & WLS3 & WLS1 & WLS2 & WLS3 & WLS4 \\
\hline i1 & 0.055 & 0.242 & 0.093 & 0.027 & 0.246 & 0.075 & 0.362 & 0.158 & -0.196 \\
\hline i2 & 0.373 & 0.299 & 0.352 & 0.149 & 0.200 & 0.412 & 0.318 & 0.138 & -0.090 \\
\hline i3 & 0.253 & 0.293 & 0.645 & -0.265 & 0.063 & 0.337 & 0.306 & -0.169 & -0.181 \\
\hline i4 & 0.442 & 0.369 & 0.486 & 0.108 & 0.275 & 0.493 & 0.311 & -0.059 & 0.095 \\
\hline i5 & 0.633 & 0.279 & 0.755 & 0.030 & 0.052 & 0.696 & 0.175 & -0.212 & 0.025 \\
\hline i6 & 0.775 & 0.120 & 0.617 & 0.291 & -0.007 & 0.799 & 0.032 & -0.072 & 0.095 \\
\hline i7 & 0.676 & 0.178 & 0.632 & 0.175 & 0.027 & 0.723 & 0.173 & 0.044 & -0.122 \\
\hline i8 & 0.658 & 0.089 & 0.567 & 0.191 & -0.015 & 0.693 & 0.118 & -0.034 & -0.155 \\
\hline i9 & -0.042 & 0.251 & -0.267 & 0.273 & 0.347 & -0.082 & 0.147 & 0.002 & 0.554 \\
\hline $\mathrm{i} 10$ & 0.678 & 0.046 & 0.595 & 0.177 & -0.073 & 0.704 & 0.002 & -0.134 & -0.008 \\
\hline i11 & 0.772 & -0.056 & 0.236 & 0.594 & 0.006 & 0.741 & -0.004 & 0.157 & -0.072 \\
\hline i12 & 0.798 & -0.121 & -0.005 & 0.871 & 0.003 & 0.755 & -0.140 & 0.090 & 0.138 \\
\hline i13 & 0.767 & -0.151 & 0.069 & 0.727 & -0.024 & 0.710 & -0.146 & 0.160 & 0.096 \\
\hline i14 & -0.339 & 0.376 & -0.288 & -0.038 & 0.545 & -0.362 & 0.431 & 0.056 & 0.204 \\
\hline i15 & 0.049 & 0.534 & 0.203 & -0.044 & 0.730 & 0.056 & 0.635 & 0.074 & 0.187 \\
\hline i16 & 0.252 & 0.242 & -0.081 & 0.420 & 0.297 & 0.233 & 0.080 & 0.013 & 0.607 \\
\hline i17 & 0.769 & -0.137 & 0.211 & 0.601 & -0.083 & 0.743 & -0.176 & 0.044 & 0.102 \\
\hline i18 & 0.084 & 0.020 & -0.163 & 0.252 & 0.108 & 0.015 & 0.055 & 0.852 & 0.000 \\
\hline
\end{tabular}

Table 4. Correlations between factors in the three-factor EFA model

\begin{tabular}{|c|c|c|c|c|c|c|c|c|}
\hline \multicolumn{4}{|c|}{ Three-factors } & \multicolumn{5}{|c|}{ Four-factors } \\
\hline & WLS1 & WLS2 & WLS3 & & WLS1 & WLS2 & WLS3 & WLS4 \\
\hline WLS1 & 1.00 & & & WLS1 & 1.00 & & & \\
\hline WLS2 & 0.63 & 1.00 & & WLS2 & 0.19 & 1.00 & & \\
\hline WLS3 & 0.16 & 0.05 & 1.00 & WLS3 & 0.07 & -0.05 & 1.00 & \\
\hline & & & & WLS4 & 0.14 & 0.06 & 0.12 & 1.00 \\
\hline
\end{tabular}

\section{CONCLUSION}

There are many questions left open. Superficially, it appears that the teachers who responded to the survey prefer teacher-centered approaches over student centered approaches. It also appears that a range of approaches that, in our judgement, have the potential to promote active learning are used infrequently. The question about whether Norwegian students would benefit from more exposure to approaches that have the potential to promote active learning cannot be addressed from this survey alone. A better, more representative sample is required. We can hypothesise, on the basis of international evidence and the thin evidence we have presented above that it is possible to stimulate students' to be more active learners and that such is likely to improve their performance in mathematics.

\section{REFERENCES}

Apkarian, N. et al. (2020). Assessing the uptake of research based instructional strategies by postsecondary mathematics. Proceedings of the 22nd Annual Conference of the Special interest Group of the Mathematical Association of America on Research in Undergraduate Mathematics Education, (pp. 18-27). Retrieved from http://sigmaa.maa.org/rume/RUME23.pdf October 7, 2020.

Enberg, K., Steen, I. H., \& Ellingsen, S. (2019). Me, myself and I - Teachers' self-motivation and sense of responsibility determine the use of active learning methods. In R. Lyng \& M. M. Jakobsen (Eds.), Proceedings of MNT-konferansen 2019, 28.-29. mars, (pp. 55-59) Tromsø.

Freeman S. et al. (2014). "Active learning increases student performance in science, engineering, and mathematics." PNAS 111 (23): 8410-8415.

Laursen, S. L. \& Rasmussen, C. (2019). I on the Prize: Inquiry approaches in undergraduate mathematics. International Journal of Research in Undergraduate Mathematics Education 5, 129-146

Rasmussen, C. Apkarian, N., Ellise, H., Johnson, E., Larsen, S., Bressoud, D., \& the Progress through Calculus team (2019). Characteristics of Precalculus through Calculus 2 programs: Insights from a national census survey. Journal for Research in Mathematics Education 50(1), 24-37. 